\title{
Valor construccional y prototipicidad de metonimias para aconsejar. Contra una lectura fuerte de la tesis fundacional de la pragmática lingüística ${ }^{1}$
}

Constructional value and prototypicality of metonymies for advising. Against a strong reading of the foundational thesis of pragmalinguistics

\section{Claudia Muñoz Tobar \\ Universidad de Concepción \\ Chile}

\section{Wilfredo Quezada Pulido}

Universidad de Santiago de Chile Chile

\section{(C) $\underset{\mathrm{BY}}{(\mathrm{ND}}$}

Claudia Muñoz Tobar: Departamento de Filosofía, Facultad de Humanidades y Arte, Universidad de Concepción, Chile. | Correo electrónico: claumuno@udec.cl

Wilfredo Quezada Pulido: Departamento de Filosofía, Facultad de Humanidades, Universidad de Santiago de Chile. | Correo electrónico: wilfredo.quezada@usach.cl 


\section{Resumen}

En este trabajo analizamos los datos recogidos en una prueba exploratoria, destinada a establecer una medida de la fuerza ilocutiva de consejo de 15 enunciados. El análisis muestra que la lectura pragmática de las expresiones depende en gran parte de sus rasgos construccionales, como han propuesto Pérez (2001), Ruiz de Mendoza y Baicchi (2007) y Del Campo (2012, 2013), en el marco del estudio de las construcciones ilocutivas. En consecuencia, no es válido sostener una interpretación fuerte de la tesis de Wittgenstein (1953) de que "el significado es el uso en el lenguaje”. Esta interpretación, rechazada, entre otros, por Searle (1969), impone un límite insuperable a las pretensiones explicativas de la pragmática lingüística, lo que no se condice con nuestros resultados.

Palabras clave: acto de habla; consejo; metonimia; construcción ilocutiva; pragmática.

\section{Abstract}

In this paper we analyze the data collected in an exploratory test, designed to establish a measure of the advise illocutionary force of the 15 utterances. The analysis shows that the pragmatic reading of expressions depends largely on their constructional features, as Pérez (2001), Ruiz de Mendoza \& Baicchi (2007) and Del Campo (2012, 2013) have proposed within the framework of the study on illocutionary constructions. Consequently, it is not valid to state a strong interpretation of Wittgenstein's (1953) thesis that "the meaning is the use in the language". This interpretation, rejected, among others, by Searle (1969), imposes an insurmountable limitation to the explanatory pretensions of pragmalinguistics, which does not correlate with our results.

Keywords: speech act; advising; metonymy; illocutionary construction; pragmatics.

1 Este trabajo es un resultado del proyecto DIUC No 212.063.010-1.0, patrocinado por la Vicerrectoría de Investigación y Desarrollo de la Universidad de Concepción. Una parte de esta investigación se inscribe, además, en el proyecto Fondecyt 1080415 del coinvestigador. 


\section{Introducción}

En una prueba aplicada a 90 hablantes de español, se les solicitó que evaluaran 15 oraciones e indicaran en qué grado les parecían útiles para realizar consejos. Las oraciones expresaban variables distintas del modelo cognitivo del acto de habla de aconsejar. Nuestro objetivo fue obtener una medida del valor construccional de los ejemplos (potencial para expresar consejos), representado en su índice de prototipicidad, sensible a los mecanismos cognitivos involucrados en la construcción de su significado ilocutivo. Nuestra base fue el enfoque metonímico de Thornburg y Panther (1997), que define los actos de habla indirectos como tipos de metonimias parte-todo, es decir, enunciados capaces de evocar el acto de habla como un todo (p. e., una petición), mediante la expresión de alguna de sus condiciones de fortuna (p. e., la habilidad del oyente de realizar la acción). En el análisis de los datos nos atuvimos al desarrollo crítico del enfoque metonímico de Pérez y Ruiz de Mendoza (2002) y a su elaboración construccional, realizada por Pérez (2001), Ruiz de Mendoza y Baicchi (2007), Ruiz de Mendoza (2011) y Del Campo (2012, 2013). Pretendíamos obtener evidencia del valor analítico y explicativo del concepto de construcción ilocutiva que estos autores han propuesto y desarrollado en los últimos años. Asimismo, buscábamos sustentar nuestra tesis de que el enfoque construccional de la ilocución es más potente que el modelo pragmático inferencialista (Searle, 1969, 1975; Grice, 1975), para enfrentar las consecuencias epistemológicas derivadas del fuerte externalismo asociado a la tesis fundacional de la pragmática lingüística, formulada por Wittgenstein (1953: \$43), de que "el significado de una palabra² es su uso en el lenguaje”. Dado que los usos, o las actividades humanas en que ocurren, son innumerables (\$23), no sería posible ofrecer modelos teóricos que den cuenta de la determinación pragmática del significado, implicada por la tesis, lo que limita las aspiraciones de la pragmática lingüística a la mera descripción de los diversos usos del lenguaje. Searle (1969), en una posición menos radical, advirtió que ciertos mecanismos léxico-gramaticales - los "dispositivos indicadores de fuerza ilocutiva” (illocutionary force indicating devices) - permitían expresar actos de habla sin necesidad de inferencias; pero las construcciones ilocutivas ofrecen más ventajas. Junto con establecer una relación más estrecha entre forma y significado, hacen posible elaborar un constructicón y dar cuenta de la polisemia construccional en el nivel ilocutivo. Las construcciones ilocutivas, en efecto, lo mismo que las del nivel léxico, se relacionan entre sí formando redes y cada una puede vincularse con varios significados, resultando su valor final de conjugar su significado con el contexto de realización. Además, una construcción ilocutiva (p. e., Te prometo que ${ }_{v p}$ ) determina la composición del resto del enunciado, pues su parte fija

2 Lo mismo se aplica a oraciones y frases. Dentro del construccionismo cognitivista, donde léxico y gramática son extremos de un continuo, esta extrapolación del mundo léxico al construccional también es perfectamente admisible. 
(Te prometo que) restringe el tipo de elementos que saturan las variables (VP debe designar un acto del hablante que el oyente desea que realice) ${ }^{3}$.

En su versión revisada del enfoque metonímico, Pérez y Ruiz de Mendoza (2002) proponen que la producción e interpretación de la fuerza indirecta debe explicarse como el resultado de la interacción conceptual, que da lugar a expresiones capaces de alcanzar un alto nivel de especialización y convencionalización ilocutiva. Así generadas, las construcciones sirven de patrones para la formulación de oraciones que encarnen y comuniquen intenciones pragmáticas específicas, sin la aplicación de reglas subyacentes o conjuntos de condiciones de formación.

No obstante el lugar central de la noción de construcción ilocutiva en nuestro trabajo, tenemos que advertir que no se trata de un estudio de gramática de construcciones. Como ya precisamos, lo que nos interesa es discutir si nuestros datos pueden explicarse satisfactoriamente, tanto desde un punto de vista pragmático como cognitivo, mediante un enfoque construccional y, de ser así, mostrar que este enfoque permite afrontar el desafío epistemológico que la interpretación fuerte de la tesis de Wittgenstein (1953) plantea a la pragmática. Autores como Dummet (1981), Winch (1958), Tomassini (1988) y Rivano (2009) asumen esta interpretación de $\$ 43$ y lo que se sigue de él, aunque ninguno, excepto Rivano (1999), se declara tan radicalmente a favor. Tomassini (1988), sin embargo, parece enfático. Reconoce que los usos se suceden unos a otros y surgen en conexión con las prácticas, por lo que no puede haber un enfoque a priori y formal que fundamente una teoría del significado aceptable, sino solo descripciones de usos lingüísticos. Dummett (1981), por su parte, propone dos interpretaciones de la tesis, ambas —al menos desde nuestra perspectiva - igualmente fuertes, pues presuponen que no hay un principio constitutivo (key concept) que vaya más allá de los usos de las emisiones en contexto (la diferencia es que en un caso nos fijamos en los fundamentos para la emisión y en el otro, en sus consecuencias). Winch (1958) asume un relativismo metodológico que piensa que fluye de la tesis wittgensteiniana y de los conceptos de seguir una regla y forma de vida. Finalmente, Rivano (2009) sostiene que no es posible un conocimiento descriptivo ni explicativo de la adecuación pragmática, pues como hablantes tenemos solo una intuición sobre lo apropiado e inapropiado, pero ninguna forma de testear sus causas.

\section{Marco teórico}

Thornburg y Panther (1997) explican la producción y comprensión del significado ilocutivo indirecto como el resultado de la operación de principios metonímicos sobre estructuras de

3 Agradecemos a Ruiz de Mendoza esta advertencia acerca de las ventajas del enfoque construccional cognitivista sobre el de Searle, así como sus valiosos comentarios a este manuscrito. 
conocimiento llamadas escenarios de actos de habla, que resumen y organizan la experiencia cotidiana y reiterada de realizar un acto de habla específico. Consideremos el escenario para directivos, basado en las formulaciones de Thornburg y Panther (1997) y Ruiz de Mendoza y Del Campo (2012):

(i) COMPONENTE ANTES:

a.- El oyente $\mathrm{O}$ tiene la habilidad para realizar la acción $\mathrm{A}$ (habilidad de O).

b.- El hablante $\mathrm{H}$ desea que $\mathrm{O}$ realice $\mathrm{A}$ (motivación de $\mathrm{H}$ ).

(ii) COMPONENTE CENTRAL:

a.- H pone O bajo una obligación de realizar A.

b.- $O$ está obligado a realizar A.

(iii) COMPONENTE DESPUÉS:

a.- $O$ realizará $A$ (o no realizará $A$ ).

b.- Habrá una respuesta emocional en $\mathrm{H}$.

Cada fase (o componente) de un escenario de acto de habla puede seleccionarse para cumplir una función metonímica en los enunciados que, de esta manera, pueden evocar el escenario como un todo y comunicar la fuerza ilocutiva correspondiente. Los enunciados resultantes (como 1-3, abajo) reciben el nombre de metonimias ilocutivas (Thornburg y Panther, 1997; Panther y Thornburg, 2007). Según este enfoque, que Ilamamos metonímico, la metonimia conceptual (Johnson, 1991 [1987]; Barcelona, 2003; Lakoff y Johnson, 2004 [1980]) sirve de guía en el trabajo inferencial que conduce a la comprensión de la fuerza indirecta expresada por el hablante. Como se observa, las fases del escenario para directivos (arriba) se corresponden, en gran parte, con las condiciones de fortuna de los actos ilocutivos directivos de Searle (1969). En los ejemplos 1-3, una de las condiciones de fortuna (o fase del escenario) cumple una función metonímica (los elementos instanciadores se destacan en negrillas):

(1) ¿Puedes ayudarme a cargar la maleta? (petición).

(2) Quiero que te laves las manos (orden).

(3) Vas a ordenar tu pieza (orden).

La condición de la habilidad de O evoca en 1 una petición; 2 expresa una orden a través de la función metonímica de la motivación de H, y también 3, pero mediante la referencia al resultado del acto de habla, es decir, al parámetro O realizará A. De esta forma, el enfoque metonímico da un giro cognitivo a las generalizaciones mediante las cuales Searle (1975) da cuenta de las fórmulas para realizar actos de habla indirectos directivos y comisivos, y de las inferencias que conducen a su fuerza ilocutiva. En su artículo, Searle (1975) señala que los enunciados para realizar ilocutivos indirectos (similares a 1-3) suelen expresar literalmente preguntas o 
afirmaciones acerca de alguna de las condiciones de fortuna del acto de habla que se intenta realizar (ilocutivo indirecto o primario) y, a partir de una serie de ejemplos, extrae 9 generalizaciones que resumen distintas versiones de esta misma estrategia (Searle, 1975: 72, 81-82). La ventaja del enfoque metonímico sobre el modelo de Searle es su economía cognitiva, puesto que los principios metonímicos por los cuales las condiciones expresivas activan un escenario, almacenado en la memoria a largo plazo (Pérez y Ruiz de Mendoza, 2002: 262), funcionan como patrones para trazar inferencias y calcular con gran rapidez la intención ilocutiva del emisor.

Pese a sus ventajas, los lingüistas españoles antes citados consideran que el enfoque metonímico es insuficiente para dar cuenta de la producción e interpretación de actos de habla indirectos, pues la mera evocación de la estructura escénica no explica las diferencias en el grado de fuerza ilocutiva transmitidas por enunciados que son funciones metonímicas de un mismo componente (Pérez y Ruiz de Mendoza, 2002), como las que se dan entre los pares de enunciados con fuerza directiva 4-6', que instancian la misma fase del escenario para directivos:

(4) ¿Puedes ayudarme a cargar la maleta?

(4') ¿Podrías ayudarme a cargar la maleta?

(5) Quiero que vengas esta tarde.

(5)' Quisiera que vinieras esta tarde.

(6) Tienes que lavarte las manos.

(6’) Tendrías que lavarte las manos.

Para subsanar estas dificultades, Pérez y Ruiz de Mendoza (2002) integran los escenarios de actos de habla de cada subcategoría ilocutiva en Modelos Cognitivos Idealizados proposicionales, uno de los tipos de estructuras de organización de conocimiento introducidas clásicamente por Lakoff (1987), y proponen mecanismos de conceptualización que dan cuenta de estas diferencias. Un modelo cognitivo idealizado de acto de habla incluye, junto a la información contenida en el escenario correspondiente, las variables de coste-beneficio, opcionalidad y poder social (las escalas pragmáticas de Leech, 1997 [1983]), involucradas en la construcción del significado ilocutivo directivo y comisivo. Por ejemplo, el escenario de directivos queda integrado en la estructura del modelo cognitivo de peticiones de Pérez y Ruiz de Mendoza (2002: 264) de la siguiente manera:

Escenario de directivos de Thornburg y Panther más:

(i) A representa un costo para $\mathrm{O}$ y un beneficio para $\mathrm{H}$.

(ii) Alta opcionalidad (cortesía).

(iii) La relación de poder entre $\mathrm{H}$ y O es irrelevante.

La interpretación del sentido ilocutivo dependerá, entonces, de la fase del escenario evocada, de su relevancia para definir la categoría, del número de parámetros activados y del grado en 
que una expresión instancia las condiciones escalares del modelo cognitivo ilocutivo (Pérez y Ruiz de Mendoza, 2002). Posteriormente, Ruiz de Mendoza (2007) distingue entre modelos cognitivos de bajo y alto nivel, siendo sobre estos últimos (entre ellos, los modelos cognitivos ilocutivos) que operaría la metonimia conceptual (nos referiremos a esto más adelante).

Volvamos ahora a nuestros ejemplos. Es claro que 4', 5' y 6' tienen una lectura menos directiva que sus respectivos pares 4,5 y 6 , lo que no tiene que ver con el componente del escenario ilocutivo instanciado, que es el mismo, sino con variaciones en el modo y tiempo de los verbos modales (poder, querer y tener que), que permiten expresar distintos valores de los parámetros escalares de obligatoriedad, opcionalidad, coste-beneficio y directividad. En el par 4/4' la variación temporal (puedes-podrías) produce diferencias en el nivel de cercanía conceptual entre el oyente 0 y su habilidad de realizar A, que es mayor en 4', donde el verbo se expresa en condicional (podrías). Este mecanismo, sumado a la formulación interrogativa y al uso reflexivo del verbo principal (ayudar + me), que activa el parámetro del beneficio del hablante, aumenta la opcionalidad para el destinatario y hace de 4' un enunciado menos directivo. En el par 5/5', la variación de modo (quiero-quisiera) refleja niveles distintos de deseo de que 0 realice A - en 5 (presente) es mayor que en 5' (subjuntivo) - , dando lugar a niveles directamente proporcionales de directividad. En 6/6', finalmente, la variación de tiempo de los modales (tienes-tendrías) produce diferencias en el grado de obligatoriedad que transmiten. El uso del subjuntivo en 5' (quisiera) y del condicional en 6' (tendrías) se asocian, respectivamente, a niveles más bajos de deseo de $\mathrm{H}$ y de obligatoriedad para $\mathrm{O}$, que los transmitidos por 5 y 6 , donde se usa el presente de indicativo (quiero-tienes), lo que hace que la fuerza directiva de los primeros sea menor. Obsérvese que estas diferencias se relacionan con el grado de prototipicidad de las construcciones (4 y 4' son más representativos de petición que 5 y 5') y con su potencial de instanciación, que consiste en su capacidad para evocar parámetros específicos del modelo cognitivo de petición (6’ activa bajo grado de obligatoriedad). Pérez y Ruiz de Mendoza (2002: 270) proponen que la generación de expresiones con estas condiciones diferenciadoras y el cálculo de la fuerza indirecta que transmiten se debe a la interacción conceptual de los cuatro tipos de modelos cognitivos idealizados introducidos por Lakoff (1987): proposicionales, de esquemas de imagen, metonímicos y metafóricos. Por ejemplo, un esquema de distancia conceptual (Pérez y Ruiz de Mendoza, 2002), generado por una doble operación metafórica, explicaría que el subjuntivo en 5' (quisiera) transmita menor grado de directividad que el presente de indicativo en 5 (quiero). El primer paso consiste en la proyección del espacio sobre el tiempo (TIEMPO ES ESPACIO) y, luego, a partir del esquema de orientación espacial cerca-lejos (Johnson, 1991 [1987]), la distancia temporal se

4 Ruiz de Mendoza (2007) distingue los MCls operacionales de los no operacionales. Metáfora y metonimia son operativos, pues actúan sobre las condiciones semánticas de MCls no operacionales, como esquemas de imagen y modelos proposicionales. 
proyecta sobre la implicación personal o social. De esta manera, el subjuntivo permite a H crear mayor distancia y expresar, entonces, menor implicación entre él y su deseo de que 0 haga A. En consecuencia, el esquema de distancia conceptual constituye la base semántica para la operación metonímica (parte-todo) que activa el modelo cognitivo de petición y dirige la inferencia. Sin embargo, como lo han hecho notar posteriormente los mismos autores, la interpretación del significado ilocutivo no siempre surge de procesos inferenciales guiados metonímicamente, sino que depende muchas veces del nivel de convencionalización de las expresiones. En los últimos años, este aspecto ha sido considerado en el estudio de la ilocución por Ruiz de Mendoza y Baicchi (2007), Baicchi y Ruiz de Mendoza (2010), Pérez y Ruiz de Mendoza (2011) y Del Campo (2013), dentro del marco del Modelo Léxico Construccional (Ruiz de Mendoza y Mairal, 2008; Ruiz de Mendoza y Gonzálvez, 2010; Del Campo, 2013), a través del concepto de construcción ilocutiva, lo que ha permitido integrar la teoría del acto de habla en el campo de estudio de la Gramática de Construcciones (Fillmore, 1985, 1988; Langacker, 1987; Fillmore y otros, 1988; Goldberg, 1995, 2003; Croft, 2001, entre otros).

\subsection{Enfoque construccional de la ilocución}

Cuando las expresiones generadas mediante interacción conceptual alcanzan altos niveles de convencionalización, adquieren el estatus de construcciones ilocutivas, que son formas altamente especializadas para la transmisión de tipos específicos de actos de habla (Del Campo, 2012). En el Modelo Léxico Construccional, las construcciones ilocutivas pertenecen al tipo de las idiomáticas5, constituidas por un elemento fijo (o idiomático) y estructuras argumentales como elementos variables (7-9, abajo). En 7, Will you es el elemento fijo de la construcción, mientras VP es el variable, que puede completarse mediante una frase verbal, como "help me", en 10 (tomado de Pérez, 2001), para expresar una petición, funcionando, de esta manera, como un patrón para generar expresiones con este valor ilocutivo. Las construcciones 8 y 9 tienen instancias en 11 y 12 , respectivamente (los ejemplos 7, 8 y 11 pertenecen a Del Campo, 2013):
(7) Will you
(8) Consider $X_{v p} \quad$ (paraconsejos).
(9) “¿Qué tal si vp?" (para ofertas).
(10) "Will you help me?"

5 Las construcciones argumentales, por otro lado, unen léxico y sintaxis de acuerdo al número y tipo de argumentos requeridos por las unidades léxicas (Del Campo, 2013: 321). 
(11) "Consider getting a housekeeper twice monthly if the budget allows".

(12) ¿Qué tal si dejamos hasta aquí el trabajo y nos vamos a tomar un café?

La interpretación de los enunciados que realizan una base construccional ilocutiva es altamente independiente del contexto, pero esto no debe entenderse en términos de un concepto fuerte de literalidad, sino más bien bajo la idea de grados de convencionalización (Pérez, 2001). Mientras más convencionalizado es el uso de una expresión para realizar un tipo de acto de habla, menor es la necesidad del contexto y el número de inferencias para atribuirle un significado ilocutivo específico y, por lo tanto, mayor su grado de especialización. Un aspecto relevante de este modelo, desde nuestra perspectiva, es que la descripción de las construcciones ilocutivas como patrones para la generación de expresiones es compatible con la innumerabilidad de las formas de realización de la fuerza pragmática, pero no con la lectura fuerte de la tesis de Wittgenstein de que esto representa un límite para cualquier pretensión explicativa. Desde el enfoque construccional, el sentido pragmático se construye mediante la instanciación de las condiciones semánticas (parámetros de modelos cognitivos) de las construcciones ilocutivas por la operación de mecanismos cognitivos de construcción (como las interpretaciones metonímicas y metafóricas). Esto tiene consecuencias en dos sentidos. Por un lado, aunque una expresión, considerada fuera de un contexto particular de uso, tenga una variedad amplia de posibilidades significativas, como sostiene Searle (1992), parte de estas posibilidades dependerán de la forma utilizada, es decir, de la medida en que esta sea capaz de evocar un concepto ilocutivo específico, lo que, junto con significar variaciones en la valoración de su utilidad para transmitir la intención pragmática (potencial de adecuación), supone ciertas restricciones de interpretación. Por otro lado, dada una intención ilocutiva particular, las posibilidades expresivas, aunque amplias, no pueden ser cumplidas con la misma eficiencia por las expresiones. La dimensión construccional permite dar cuenta de las diferencias que las expresiones lingüísticas presentan en estas dos direcciones, pues, si se acepta que la asignación de un sentido ilocutivo específico a las oraciones — con independencia de que puedan expresar distintos actos de habla en contextos adecuados-depende en una buena medida de sus valores construccionales (su potencial de activar parámetros del modelo cognitivo), esas diferencias deberían vincularse de manera consistente con los mecanismos de construcción asociados a las oraciones, como por ejemplo con variaciones en los elementos que dentro de ellas instancian parámetros de mayor o menor especificidad y relevancia para definir la categoría ilocutiva del caso, y con el grado en que lo hacen. Consideramos que esta hipótesis hace justicia a la intuición de que los hablantes, al comunicar la fuerza ilocutiva, realizan un ajuste entre las posibilidades expresivas con que cuentan y la cantidad de información ofrecida por el contexto, pues, como señala Del Campo (2012), las oraciones generadas a partir de una base construccional (como 10-12, más arriba) pueden explicitar en grados diversos los parámetros construccionales, dependiendo del propósito comunicativo del hablante o de la información contextual disponible. 


\subsection{Modelos cognitivos y construcción ilocutiva}

Junto con reducir la cantidad de inferencias necesarias para interpretar actos de habla, las construcciones ilocutivas son interesantes debido a su condición de patrones para la expresividad. En tanto constituyen "sistemas de instanciaciones y parametrizaciones de condiciones semánticas de modelos cognitivos genéricos” (Del Campo, 2013: 320), almacenados en nuestra memoria a largo plazo, es decir, dado el nivel de especialización funcional del que depende su capacidad para activar conjuntos de esas condiciones (Del Campo, 2012; Ruiz de Mendoza y Del Campo, 2012), las construcciones ilocutivas nos permiten (re)construir un significado ilocutivo para enunciados concretos.

Dentro del Modelo Léxico Construccional, que ha sido el marco para el estudio cognitivo de la ilocución, se distinguen distintos niveles de organización conceptual jerárquicamente relacionados (Ruiz de Mendoza, 2007). En particular, los modelos cognitivos de actos de habla, el modelo de coste-beneficio (Ruiz de Mendoza y Baicchi, 2007) ${ }^{6}$ y los escenarios ilocutivos son tipos de Modelos Cognitivos Situacionales de Alto Nivel, entendiendo por esto que recogen la estructura genérica común a grupos de Modelos Cognitivos Situacionales de Bajo Nivel (Del Campo, 2012). Los modelos situacionales de bajo nivel capturan aspectos de interacciones cotidianas procesuales y estables (Ruiz de Mendoza y Galera, 2012), como tomar un taxi, ir a un restaurante, dar una clase, ir a la consulta de un médico o comprar en el kiosco del barrio (los scripts de Schank y Abelson, 1977). En relación con los consejos, los modelos de este tipo incluyen el conocimiento de diferentes situaciones y formas en que decimos a otros lo que deberían hacer para modificar estados de cosas que los afectan negativamente (Del Campo, 2012), mientras que el modelo situacional de alto nivel, que recoge la semántica del consejo, se estructura sobre la base común de esos modelos de nivel más básico. El siguiente es el modelo situacional de alto nivel para consejos formulado por Del Campo (2012), cuyos parámetros (Pa-Pf) utilizaremos en el análisis de una parte de nuestros resultados:

(Pa) B parece involucrado en una situación negativa SN.

(Pb) A cree que un curso de acción CA ayudaría a B a cambiar SN.

(Pc) A hace consciente a B de CA.

(Pd) A hace consciente a B del potencial beneficio de CA.

6 El modelo cognitivo idealizado de Coste-Beneficio recoge 11 convenciones culturales que obligan a las personas a ayudar a otras, si pueden hacerlo. Estas tienen también realización lingüística. 
(Pe) B puede estar dispuesto a obtener el beneficio.

(Pf) B puede tomar el CA propuesto.

La presencia del parámetro del beneficio de 0 en el modelo cognitivo de consejos obedece a la necesidad de incorporar convenciones culturales contenidas en el modelo de coste-beneficio (Ruiz de Mendoza y Baicchi, 2007) que son importantes en la comprensión de ilocutivos directivos y comisivos (Pérez y Ruiz de Mendoza, 2002), en especial las tres siguientes: a) si es manifiesto para A que un estado de cosas particular no es beneficioso para B, y si A tiene la capacidad de cambiar este estado de cosas, entonces A debería hacerlo; b) si es manifiesto para A que un estado de cosas potencial no es beneficioso para B, entonces no se espera que A lo lleve a cabo; y c) si es manifiesto para A que un estado de cosas potencial es beneficioso para B, entonces se espera que A lo lleve a cabo. La primera convención se aplica particularmente en la interpretación de consejos, porque es la que mueve al hablante a intervenir. Además, da cuenta de la alta opcionalidad que caracteriza a este acto de habla (el destinatario es totalmente libre de realizar la acción), basada, según lo explica muy bien Del Campo (2013), en el hecho de que el participante $B$ (destinatario en los consejos) es tanto el agente de la acción como su beneficiario y, por consiguiente, el único a quien afecta la decisión. El modelo situacional de alto nivel para consejos se apoya bastante más explícitamente en esta convención, que también puede ser activada por las expresiones.

Dependiendo del tipo particular de actos de habla, las construcciones ilocutivas (como Consider $X_{v p}$ para aconsejar) tienen unas condiciones de sentido determinadas, lo que define su especialización (Del Campo, 2013). El significado ilocutivo de una expresión procede del acceso metonímico que proporciona a las condiciones de sentido de la construcción que le sirve de base, representadas por las variables del modelo situacional de alto nivel. Más específicamente, ciertos elementos en las expresiones ofrecen puntos de acceso metonímico a una parte relevante del modelo cognitivo (Del Campo, 2012) que da anclaje semántico a la construcción ilocutiva base. El nivel de especialización y las posibilidades de convencionalización tienen que ver con el hecho de que las condiciones de sentido pueden ser activadas en distinto grado por las expresiones. Por ejemplo, la construcción 13 (abajo), a diferencia de 14, instancia un alto grado de obligatoriedad para órdenes, lo que daría cuenta de su mayor nivel de convencionalización en contextos jerárquicos. En español contamos también con las construcciones 15 y 16, cuyas instancias en 15 a y 16 a son convencionales para peticiones:

(11) Consider getting a housekeeper twice monthly if the budget allows.

(13) Tienes que tener lista la tarea cuando yo vuelva.

(14) Tendrías que tener lista la tarea cuando yo vuelva.

(15) ¿Me ayudarías con ${ }_{N P}$ ? 
(16) ¿Me ayudarías ${ }_{v p}$ ?

(15a) ¿Me ayudarías con unas monedas?

(16a) ¿Me ayudarías a bajar esos libros?

Por otra parte, 1 ( (reproducido arriba) realiza la construcción Consider $X_{v p}$ e instancia dos de sus condiciones para consejos: getting a housekeeper twice monthly expresa un estado de cosas que podría solucionar un problema del destinatario (Pc del modelo situacional), mientras que if the budget allows instancia la condición de la habilidad de $O$ (modelo cognitivo de consejos). Dado que los elementos fijos y las variables de las construcciones ilocutivas pueden instanciar parámetros del modelo cognitivo, una misma construcción puede activar varias condiciones de sentido, lo que aumenta su especificidad y su potencial de instanciación. Esto implica no solo una amplia variedad de realizaciones expresivas para un mismo acto de habla, sino también la posibilidad de que se incorporen más patrones al acopio construccional.

\section{Metodología}

No es posible explicar analíticamente por qué una construcción alcanza el nivel de uso y convencionalización que la convierte en una construcción ilocutiva, pero podemos establecer las características construccionales que favorecen este proceso y que explican por qué algunas expresiones pueden transmitir mejor que otras un determinado acto de habla. Dado que un planteamiento central de la pragmática en Lingüística Cognitiva es que la prototipicidad y el estatus construccional de una oración dependen de la relación que esta guarda con la estructura genérica del ilocutivo que transmite, nos propusimos obtener evidencia mediante una prueba de prototipicidad para la categoría de consejo, que aplicamos a 90 estudiantes universitarios, todos chilenos, hablantes de español, de entre 18 y 20 años de edad.

Nuestra hipótesis era que si hacíamos variar, en un grupo de enunciados, los parámetros instanciados del modelo cognitivo idealizado de consejos (presentado abajo) y su modalidad de presentación, de tal manera que los enunciados expresaran parámetros de diversa relevancia y representaran distintos mecanismos de construcción, encontraríamos diferencias en el potencial para la realización de consejos asignado a cada uno de ellos por el grupo de informantes. Estas diferencias revelarían valores construccionales probables para los ejemplos y, en este sentido, su potencial de adecuación pragmática, es decir, una medida de su utilidad y pertinencia para realizar consejos en un contexto ideal. De manera más específica, si la capacidad de las expresiones para transmitir un sentido ilocutivo particular depende, según nuestra hipótesis, de la cantidad, relevancia y grado de manifestación de los parámetros del modelo cognitivo que pueden activar (potencial de instanciación), entonces, las expresiones que con mayor consistencia fueran evaluadas como formulaciones claras de consejos 
por el grupo de informantes, deberían expresar parámetros de mayor relevancia, en mayor cantidad, o bien en el grado de manifestación definido por el modelo cognitivo de consejo, comparadas con las expresiones más dudosas. Esto evidenciaría que el sentido ilocutivo de una oración depende de su valor construccional, es decir, de su capacidad para activar, con independencia de un contexto específico, la estructura genérica que define el acto de habla. La independencia del contexto no se entiende aquí como un indicador de literalidad, en el sentido fuerte que esta tiene en la teoría del acto de habla, pues, dentro del enfoque construccional, el valor ilocutivo final de una expresión surgirá de combinar sus condiciones construccionales y el contexto de uso. En la formulación de los ejemplos usamos el modelo cognitivo de consejos siguiente, basado en el modelo de Pérez y Ruiz de Mendoza (2002):

(i) COMPONENTE ANTES del EAH Directivos:

a.- $O$ tiene habilidad para realizar $A$.

b.- $\mathrm{H}$ desea que $\mathrm{O}$ realice $\mathrm{A}$.

(ii) COMPONENTE CENTRAL del EAH Directivos:

a.- H pone a O bajo una obligación (más o menos fuerte) de realizar A.

b.- $O$ está obligado a realizar $A$.

(iii) COMPONENTE DESPUÉS del EAH Directivos:

a.- O realizará A (o no realizará A).

b.- Habrá una respuesta emocional en $\mathrm{H}$.

(iv) A representa un beneficio para $\mathrm{O}$ (y es neutro para $\mathrm{H}$ ).

(v) Alta opcionalidad (cortesía).

(vi) La relación de poder entre H y O es irrelevante.

En la prueba se presentaron 15 ejemplos que instanciaban distintos parámetros de este modelo. Para expresar su instanciación se usaron verbos modales (puedes, tienes que, debes, quiero) y frases adverbiales y de sujeto (es mejor que, te conviene que), mientras que para producir diferencias en el grado de manifestación de las condiciones con sentido escalar (obligatoriedad y opcionalidad) se recurrió a cambios en el tiempo y modo de los verbos modales (debes - deberías, tienes que - tendrías que), que introdujeran distintos niveles de distancia conceptual. A fin de obtener el índice de representatividad de los ejemplos, los participantes debían evaluar su pertenencia a la categoría de consejo en una escala de 1 a 7 . Un índice cercano a 7 indicaría que un al to número de encuestados consideró el enunciado como un caso claro de este acto de habla.

Previamente, para establecer qué condiciones de la categoría eran más importantes para los informantes y verificar su presencia en el modelo cognitivo, se les pidió que redactaran una breve definición de "aconsejar". Luego, separamos las condiciones mencionadas en seis 
grupos, registramos el número de menciones de cada una y obtuvimos el siguiente ordenamiento de su relevancia: beneficio de 0 (56 menciones), opcionalidad (40 menciones), 0 es afectado por una situación problemática (18 menciones), H intenta que O haga A (17 menciones), empatía del consejo (1 mención) y sabiduría de H (1 mención).

\section{Resultados y discusión}

La tabla 1 muestra los índices de representatividad (IR) obtenidos, en orden descendente. Los ejemplos conservan el número asignado a cada uno en la prueba. También se indican los resultados según el área de estudio de los informantes (Inf): no humanística, humanidades, artes y trabajo social. Dividimos los índices de representatividad en tres niveles de prototipicidad (columna 8) y señalamos el parámetro del modelo de consejos (MCl) para el cual el enunciado ofrece un punto de acceso (columna 2).

Iniciaremos la discusión de los resultados con los ejemplos que instancian fases del escenario de directivos de Thornburg y Panther (1997), incluido en el modelo cognitivo de consejos, cuyos parámetros por enunciado se señalan en la segunda columna de la tabla I (lo mismo que en las tablas 2-4, más adelante).

\subsection{Metonimias de ANTES y de CENTRO}

Como se observa en la tabla 2 (abajo), dentro del grupo de metonimias de la fase ANTES, los ejemplos que expresan el deseo de $\mathrm{H}$ de que O haga A (2 y 9) obtuvieron los índices más bajos, lo que se condice con el hecho de que el estado de cosas predicado satisface una necesidad del emisor y representa, por tanto, un costo para el destinatario y no un beneficio. Esto es relevante, porque el beneficio de o no solo forma parte del significado básico de consejo (Del Campo, 2012), sino que mostró ser el atributo más recurrente en las definiciones de los informantes. El enunciado 1 , que instancia la habilidad de 0 , obtuvo el índice de representatividad más destacado del grupo, lo que atribuimos a la alta opcionalidad que transmite.

Los ejemplos en la tabla 3 (abajo) son metonimias del componente CENTRAL (obligatoriedad). Los verbos modales "deber" y "tener que” funcionan en ellos como puntos de acceso al escenario de directivos, activando este parámetro, y sus variaciones en modo y tiempo permiten expresar grados diferentes de instanciación.

Los condicionales "deberías” y "tendrías que” en 7, 4 y 13, así como los elementos disposicionales "me parece que" y "creo que" en 4 y 6, sugieren una lectura menos directiva, lo que es coherente con los índices más altos de estos cuatro ejemplos. En cambio, 5 y 14, que no 


\section{TABLA 1}

Resumen de los resultados de la prueba

\begin{tabular}{|c|c|c|c|c|c|c|c|}
\hline \multirow{2}{*}{$\begin{array}{l}\text { EJEMPLOS DE } \\
\text { LA PRUEBA }\end{array}$} & \multirow{2}{*}{$\begin{array}{l}\text { PARÁMETRO } \\
\text { DEL MCI }\end{array}$} & $\begin{array}{l}\text { NO HUM } \\
18 \text { INF } \\
\end{array}$ & $\begin{array}{l}\text { HUM } \\
39 \text { INF } \\
\end{array}$ & $\begin{array}{l}\text { A PLÁST } \\
19 \text { INF }\end{array}$ & $\begin{array}{l}\text { T SOCIAL } \\
14 \text { INF } \\
\end{array}$ & $\begin{array}{l}\text { TODOS } \\
90 \text { INF }\end{array}$ & \multirow{2}{*}{$\begin{array}{c}\text { NIVELES DE } \\
\text { PROTOTIPICIDAD }\end{array}$} \\
\hline & & IR & IR & IR & IR & IR & \\
\hline $\begin{array}{l}\text { 3. Es bueno para } \\
\text { ti que viajes }\end{array}$ & iv BENEFICIO & 5,83 & 5,79 & 5,74 & 5,79 & 5,59 & \multirow{3}{*}{$\begin{array}{l}\text { Representativos } \\
\text { REPR }\end{array}$} \\
\hline $\begin{array}{l}\text { 15. Creo que te } \\
\text { conviene que viajes }\end{array}$ & iv BENEFICIO & 6,22 & 5,92 & 4,84 & 5,93 & 5,56 & \\
\hline $\begin{array}{l}\text { 12. Es mejor } \\
\text { que viajes }\end{array}$ & iv BENEFICIO & 6,28 & 4,79 & 4,68 & 5,64 & 5,00 & \\
\hline 7. Deberías viajar & ii-b CENTRO & 6,06 & 4,74 & 4,37 & 4,79 & 4,71 & \multirow{6}{*}{$\begin{array}{l}\text { Medianamente } \\
\text { representativos } \\
\text { M-REPR }\end{array}$} \\
\hline $\begin{array}{l}\text { 4. Me parece que } \\
\text { deberías viajar }\end{array}$ & ii-b CENTRO & 5,89 & 4,38 & 4,00 & 5,29 & 4,54 & \\
\hline $\begin{array}{l}\text { 11. Ese viaje no } \\
\text { te hará bien }\end{array}$ & iv BENEFICIO & 5,72 & 4,90 & 3,37 & 4,93 & 4,44 & \\
\hline $\begin{array}{l}\text { 1. Podrías viajar } \\
\text { otro día }\end{array}$ & i-a ANTES & 5,33 & 4,38 & 4,21 & 4,43 & 4,34 & \\
\hline $\begin{array}{l}\text { 6. Creo que tienes } \\
\text { que viajar }\end{array}$ & ii-b CENTRO & 5,44 & 4,08 & 4,00 & 4,07 & 4,13 & \\
\hline 8. Yo que tú, viajaría & - & 5,11 & 4,44 & 3,89 & 3,64 & 4,13 & \\
\hline $\begin{array}{l}\text { 13. Tendrías } \\
\text { que viajar }\end{array}$ & ii-b CENTRO & 4,39 & 3,77 & 3,00 & 4,43 & 3,63 & \multirow{6}{*}{$\begin{array}{c}\text { No } \\
\text { representativos } \\
\text { NO-REPR }\end{array}$} \\
\hline 10. Viaja no más & iii-a DESPUÉS & 3,67 & 3,46 & 3,16 & 3,00 & 3,17 & \\
\hline $\begin{array}{l}\text { 2. Preferiría que } \\
\text { no viajaras }\end{array}$ & i-b ANTES & 4,06 & 2,79 & 2,00 & 2,64 & 2,66 & \\
\hline $\begin{array}{l}\text { 9. Me gustaría } \\
\text { que no viajaras }\end{array}$ & i-b ANTES & 4,39 & 2,62 & 2,00 & 2,29 & 2,59 & \\
\hline 5. Debes viajar & ii-b CENTRO & 2,17 & 2,67 & 2,00 & 2,86 & 2,26 & \\
\hline 14. Tienes que viajar & ii-b CENTRO & 3,00 & 2,56 & 1,63 & 3,00 & 2,24 & \\
\hline
\end{tabular}

\section{TABLA 2}

Metonimias del componente ANTES

\begin{tabular}{|c|c|c|c|c|c|c|}
\hline \multirow{2}{*}{ EJEMPLOS DE LA PRUEBA } & \multirow{2}{*}{$\begin{array}{l}\text { PARÁMETRO } \\
\text { DEL MCI }\end{array}$} & NO-HUM. & HUM. & A. PLÁST. & T. SOCIAL & TODOS \\
\hline & & IR & IR & IR & IR & IR \\
\hline 1. Podrías viajar otro día & $\begin{array}{l}\text { Habilidad } \\
\text { de O }\end{array}$ & 5,33 & 4,38 & 4,21 & 4,43 & 4,34 \\
\hline 2. Preferiría que no viajaras & Deseo de 0 & 4,06 & 2,79 & 2,00 & 2,64 & 2,66 \\
\hline 9. Me gustaría que no viajaras & Deseo de 0 & 4,39 & 2,62 & 2,00 & 2,29 & 2,59 \\
\hline
\end{tabular}




\section{TABLA 3}

Metonimias del componente CENTRO

\begin{tabular}{llccccc}
\multirow{2}{*}{ EJEMPLOS DE LA PRUEBA } & $\begin{array}{c}\text { PARÁMEtRO } \\
\text { DEL MCI }\end{array}$ & NO-HUM. & HUM. & A. PLÁST. & T. SOCIAL & TODOS \\
\cline { 3 - 7 } 7. Deberías viajar & IR & IR & IR & IR & IR \\
\hline 4. Me parece que deberías viajar & Obligatoriedad & 6,06 & 4,74 & 4,37 & 4,79 & $\mathbf{4 , 7 1}$ \\
\hline 6. Creo que tienes que viajar & Obligatoriedad & 5,44 & 4,08 & 4,00 & 4,07 & $\mathbf{4 , 1 3}$ \\
\hline 13. Tendrías que viajar & Obligatoriedad & 4,39 & 3,77 & 3,00 & 4,43 & $\mathbf{3 , 6 3}$ \\
\hline 5. Debes viajar & Obligatoriedad & 2,17 & 2,67 & 2,00 & 2,86 & $\mathbf{2 , 2 6}$ \\
\hline 14. Tienes que viajar & Obligatoriedad & 3,00 & 2,56 & $\mathbf{1 , 6 3}$ & 3,00 & $\mathbf{2 , 2 4}$ \\
\hline
\end{tabular}

cuentan con mitigadores de obligatoriedad, obtuvieron los índices más bajos de la prueba (ver tabla 1). Esto último coincide con lo esperado, porque su alta obligatoriedad está en conflicto con la condición de alta opcionalidad propia del consejo.

\subsection{Parámetros del BENEFICIO DEL OYENTE y ALTA OPCIONALIDAD}

Los ejemplos prototípicos de consejo (3, 12 y 15, en tabla 4, abajo) expresan que el curso de acción predicado (viajar) beneficia al destinatario, lo que concuerda con la importancia que los informantes asignaron al parámetro del beneficio de 0. Nótese que el ejemplo 3, cuyo índice de representatividad fue el más alto de este grupo de metonimias (y de la prueba), transmite mayor certeza de que la acción beneficia a O, comparado con 15 (con el segundo índice de la prueba), donde se usa la expresión disposicional "creo que”. Al mismo tiempo, las fórmulas "para ti" y "te conviene" en 3 y 15 enfatizan el rol de beneficiario del oyente, lo que no ocurre en 12, cuyo índice fue más bajo. Esperábamos que las expresiones 3, 12 y 15 obtuvieran índices más altos que las metonimias del deseo de H (2 y 9, en tabla 2), que se oponen (como señalamos en 4.1.) a la condición del beneficio de O. Finalmente, el ejemplo 11 expresa indirectamente esta condición, pues, al apuntar al costo de la acción, sugiere que el beneficio se conseguirá evitando realizarla. La dificultad interpretativa que esto impone y el hecho de que en la prueba la acción beneficiosa se expresó siempre en su forma positiva (viajar) puede explicar su bajo índice de representatividad dentro del grupo.

Por otra parte, para expresar grados distintos de opcionalidad, aplicamos estrategias gramaticales en los puntos de acceso a la variable de obligatoriedad (ver tabla 5, abajo). Algunas de estas estrategias generan efectos desde el interior de la estructura del punto de acceso, como el modo y tiempo de los verbos de obligatoriedad (“deberías" en 7 y "tendrías" en 13), 


\section{TABLA 4}

Metonimias del BENEFICIO del oyente

\begin{tabular}{lccccccc}
\multirow{2}{*}{ EJEMPLOS DE LA PRUEBA } & PARÁMETRO & NO-HUM. & HUM. & A. PLÁST. & T. SOCIAL & TODOS \\
\cline { 6 - 9 } & DEL MCI & IR & IR & IR & IR & IR \\
3. Es bueno para ti que viajes & Beneficio de O & 5,83 & 5,79 & 5,74 & 5,79 & $\mathbf{5 , 5 9}$ \\
\hline $\begin{array}{l}\text { 15. Creo que te conviene } \\
\text { que viajes }\end{array}$ & Beneficio de O & 6,22 & 5,92 & 4,84 & 5,93 & $\mathbf{5 , 5 6}$ \\
\hline 12. Es mejor que viajes & Beneficio de O & 6,28 & 4,79 & 4,68 & 5,64 & $\mathbf{5 , 0 0}$ \\
\hline 11. Ese viaje no te hará bien & Beneficio de O & 5,72 & 4,90 & 3,37 & 4,93 & $\mathbf{4 , 4 4}$ \\
\hline
\end{tabular}

mientras que otras lo hacen externamente, como las fórmulas disposicionales, que atenúan la obligatoriedad asociada a ellos ("me parece que" en 4 y "creo que" en 6), transmitiendo menor convicción sobre la necesidad de que O haga A. Obsérvese, otra vez en consonancia con lo reportado sobre las metonimias de CENTRO, que los índices son más altos cuando se usan estas fórmulas mitigadoras (7, 13, 4 y 6), mientras que son notoriamente más bajos cuando los verbos se presentan en presente de indicativo y sin ellas (5 y 14).

\section{TABLA 5}

Parámetro de OPCIONALIDAD

\begin{tabular}{lccccc}
\multirow{2}{*}{ EJEMPLOS DE LA PRUEBA } & NO-HUM. & HUM. & A. PLÁST. & T. SOCIAL & TODOS \\
\cline { 2 - 6 } 7. Deberías viajar & IR & IR & IR & IR & IR \\
\hline 4. Me parece que deberías viajar & 5,89 & 4,38 & 4,00 & 5,29 & $\mathbf{4 , 5 4}$ \\
\hline 6. Creo que tienes que viajar & 5,44 & 4,08 & 4,00 & 4,07 & $\mathbf{4 , 1 3}$ \\
\hline 13. Tendrías que viajar & 4,39 & 3,77 & 3,00 & 4,43 & $\mathbf{3 , 6 3}$ \\
\hline 5. Debes viajar & 2,17 & 2,67 & 2,00 & 2,86 & $\mathbf{2 , 2 6}$ \\
\hline 14. Tienes que viajar & 3,00 & 2,56 & $\mathbf{1 , 6 3}$ & 3,00 & $\mathbf{2 , 2 4}$ \\
\hline
\end{tabular}

\subsection{Parámetros del Modelo Cognitivo Situacional de Alto nivel de consejos}

El modelo situacional (MCS ${ }^{A N}$ ) para consejos de Del Campo (2012) recoge aspectos genéricos comunes a las situaciones de aconsejar, que son importantes en la comprensión de la categoría, pero que el modelo cognitivo de Pérez y Ruiz de Mendoza (2002) no incluye. En la tabla 6 (última columna) reproducimos sus parámetros. Algunos ejemplos contienen puntos de acceso a varios de ellos y casi todos activan Pc, excepto 2 y 9 (ver columnas 1 y 2 ). 


\section{TABLA 6}

Parámetros del MCS ${ }^{\mathrm{AN}}$ de consejos activados por la prueba

\begin{tabular}{|c|c|c|c|c|c|c|c|c|}
\hline \multirow{2}{*}{ EJEMPLOS DE LA PRUEBA } & \multicolumn{6}{|c|}{ PARÁMETROS DEL MCSAN } & \multirow{2}{*}{ IR } & \multirow{2}{*}{$\begin{array}{l}\text { PARÁMETROS MCSAN } \\
\text { DE CONSEJOS }\end{array}$} \\
\hline & PA & PB & PC & PD & PE & PF & & \\
\hline 3. Es bueno para ti que viajes & & & $x$ & $x$ & & & 5,59 & \multirow{2}{*}{$\begin{array}{l}\text { (Pa): B parece estar } \\
\text { envuelto en una } \\
\text { situación negativa SN. }\end{array}$} \\
\hline 15. Creo que te conviene que viajes & & $x$ & $x$ & $x$ & & & 5,56 & \\
\hline 12. Es mejor que viajes & & & $x$ & $x$ & & & 5,00 & \multirow{3}{*}{$\begin{array}{l}\text { (Pa): B parece estar } \\
\text { envuelto en una } \\
\text { situación negativa SN. }\end{array}$} \\
\hline 7. Deberías viajar & & & $x$ & & & & 4,71 & \\
\hline 4. Me parece que deberías viajar & & $x$ & $x$ & & & & 4,54 & \\
\hline 11. Ese viaje no te hará bien & & & $x$ & & & & 4,44 & \multirow{2}{*}{$\begin{array}{c}\text { (Pc): A hace consciente } \\
\text { a B de CA. }\end{array}$} \\
\hline 1. Podrías viajar otro día & & & $x$ & & & & 4,34 & \\
\hline 6. Creo que tienes que viajar & & & $x$ & & & & 4,13 & \multirow{3}{*}{$\begin{array}{c}(\mathbf{P d}) \text { : A hace } \\
\text { consciente a B } \\
\text { del potencial } \\
\text { beneficio de CA. }\end{array}$} \\
\hline 8. Yo que tú, viajaría & & & $x$ & & & & 4,13 & \\
\hline 13. Tendrías que viajar & & & $x$ & & & & 3,63 & \\
\hline 10. Viaja no más & & & $x$ & & & & 3,17 & \multirow{2}{*}{$\begin{array}{l}\text { (Pe): B puede estar } \\
\text { dispuesto a obtener } \\
\text { el beneficio de CA. }\end{array}$} \\
\hline 2. Preferiría que no viajaras & & - & - & - & - & - & 2,66 & \\
\hline 9. Me gustaría que no viajaras & & - & - & - & - & - & 2,59 & \multirow{3}{*}{$\begin{array}{l}\text { (Pf): 'B puede tomar } \\
\text { el CA propuesto'. }\end{array}$} \\
\hline 5. Debes viajar & & & $x$ & & & - & 2,26 & \\
\hline 14. Tienes que viajar & & & $x$ & & & - & 2,24 & \\
\hline
\end{tabular}

En cuanto a Pa y Pe (además de Pf, en los casos que expresan mayor opcionalidad), asumimos su activación como un efecto de la prueba, al evocar ésta el modelo. La ausencia de puntos de acceso queda compensada, además, por la conexión lógica de los parámetros en el modelo situacional. Nótese que 15 es el único ejemplo que se ajusta claramente al sentido de la creencia de $\mathrm{H}$ expresado en la condición Pb (CA podría cambiar la situación negativa), pues el emisor 'cree que' O se beneficiará al realizar CA. Los ejemplos 4 y 6 también expresan una creencia de $\mathrm{H}$ acerca de CA, pero esta se refiere a la necesidad de que O realice CA y no a la posibilidad de que beneficie a 0 , lo que sí es claro en 15, bajo el siguiente razonamiento (con Pa subentendido): dado que O está envuelto en una situación negativa (Pa), si H manifiesta que cree que CA beneficiará a O (ejemplo 15), entonces H cree que CA ayudará a O a cambiar esa situación.

Como indicamos, todos los ejemplos, excepto 2 y 9, activan Pc a través de la explicitación de CA (viajar), dado que Pc (asumiendo que la prueba evoca el modelo situacional) supone 
que CA puede modificar la situación negativa que afecta a O (Pb). Los ejemplos 2 y 9 quedan fuera, debido a que en ellos CA satisface el deseo del hablante, lo que difiere del sentido del consejo y, más específicamente, del parámetro Pb, donde CA cambia una situación negativa para O. No obstante, los enunciados activan Pc en distinta medida; 15 y 4, así como 3 y 12, lo hacen más claramente, lo que puede atribuirse al hecho de que instancian un mayor número de parámetros del modelo situacional: 15 y 4 instancian Pb y Pc, mientras que 3, 15 y 12 activan Pc y Pd (adviértase que 15 instancia los tres). La activación de un mayor número de parámetros se condice con los altos índices de representatividad de estos ejemplos (tabla 6, arriba). Por otra parte, los bajos índices de 13, 10, 5 y 14 pueden explicarse porque, al expresarse la acción en términos de una exigencia para $\mathrm{O}$, se apartan de Pf (B puede tomar CA), que apunta a la opcionalidad propia del consejo. Dado que baja obligatoriedad se corresponde con alta opcionalidad, resulta claro que esta última fue la segunda condición relevante en la estimación del valor ilocutivo de los ejemplos. Por último, los enunciados que expresan Pd (A hace consciente a B del potencial beneficio de CA) obtuvieron los índices más altos de la prueba, confirmando nuevamente que el beneficio de 0 es el parámetro más significativo.

En cuanto a los dos tipos de modelos cognitivos de consejo considerados en este trabajo, el modelo de Pérez y Ruiz de Mendoza (2002) y el modelo situacional de Del Campo (2012), es necesario señalar aspectos importantes en relación con las posibilidades de análisis que ofrece cada uno. El primero permite dar cuenta de las diferencias en prototipicidad de expresiones que realizan una misma fuerza ilocutiva, en términos de los mecanismos de interpretación involucrados en la construcción de su significado (metáfora, metonimia y esquemas de imágenes), y no solo del parámetro que cumple la función metonímica. El modelo situacional, por otra parte, resulta más apropiado para entender los procesos inferenciales que permiten captar el sentido ilocutivo. Esto se debe a que recoge en su estructura genérica los aspectos relevantes y lógicamente conectados de la situación donde el acto de habla cumple su función. Nuestro análisis de los ejemplos construidos a partir del modelo cognitivo de Pérez y Ruiz de Mendoza muestra con claridad la relación entre las propiedades semánticas de los mismos y las diferencias observadas en la valoración de su calidad de consejos, lo que se debe, fundamentalmente, a que el modelo incluye el grado de opcionalidad y obligatoriedad. Adicionalmente, las condiciones expresivas que permiten activarlas y transmitir diferencias de grado responden a la operación de la metáfora y esquemas de imágenes sobre ese material lingüístico. Tomando en cuenta lo anterior, la prueba aplicada muestra que la evocación metonímica de una misma condición del modelo cognitivo de consejos no es suficiente para explicar las diferencias en prototipicidad de las distintas expresiones que la instancian. En efecto, si estímulos lingüísticos específicos, como el modo y tiempo de verbos de obligación y expresiones disposicionales, asociados a la operación de determinados mecanismos cognitivos, son usados como puntos de acceso metonímico a una misma variable del modelo cognitivo de consejos, se obtienen resultados distintos en la evaluación de la utilidad ilocutiva de las expresiones que los contienen. En relación con el modelo situacional, nuestros 
datos pueden explicarse por la activación de las condiciones interconectadas que definen las situaciones de consejo, asociadas al sentido de los enunciados de la prueba, ya que la expresión de unas presupone la activación de otras. Si es así, las respuestas de los informantes surgen de conclusiones de razonamientos basados en la mutua relación que mantienen esas condiciones en el modelo.

\section{Conclusiones}

Consideramos que esta investigación ofrece evidencia de que la asignación del valor ilocutivo es sensible a los rasgos construccionales de los enunciados, lo que contradice la tesis externalista que limita el alcance epistemológico de la pragmática lingüísticaª En particular, se ha mostrado que los parámetros del beneficio de 0 y de alta opcionalidad se asociaron con índices de representatividad más altos. No obstante, los índices y las definiciones de los informantes muestran que el primero es el parámetro más destacado.

Como se observa en la tabla 1, la mayoría de los informantes consideró como mejores ejemplos de consejo (REPR) aquellos que instanciaban el beneficio de 0 , específicamente 3, 15 y 12 (ver tabla 6, arriba). Ahora bien, de acuerdo con el análisis basado en el modelo situacional de alto nivel, debido a que el beneficiario de la acción predicada es el mismo agente, los ejemplos que instancian el beneficio de O también expresan alta opcionalidad. Nótese que, en casi todos los ejemplos medianamente representativos (M-REPR), la expresión de un alto grado de opcionalidad se asoció a recursos mitigadores de obligatoriedad, como expresiones disposicionales y variaciones de tiempo y modo en verbos de obligación. Los ejemplos donde no se usaron estos recursos obtuvieron índices más bajos. En cuanto a los enunciados no representativos (NO-REPR), estos expresan alta obligatoriedad (5 y 14), o implican que la acción beneficia a $\mathrm{H}$ y no a O (2 y 9), o bien no explicitan si beneficia a O (13 y 10). Aunque activan parámetros que pueden vincularse a directivos, se requieren datos de contexto adicionales, que nuestra prueba no proporciona, para atribuirles el sentido de consejos, de manera que, en esta primera aproximación, donde tratamos con interpretaciones ilocutivas por defecto, su baja prototipicidad podría significar que su valor construccional para esta clase de ilocutivos es escaso. No obstante, hay que advertir que el carácter polisémico característico de las construcciones hace necesario avanzar en otras pruebas que incluyan variables de contexto, para verificar si, bajo estas condiciones, y en correspondencia con nuestra intuición como hablantes, estos enunciados podrían ser evaluados como buenos ejemplos.

7 Searle (1969) intentó mostrar que esta tesis era errónea, pero asumiendo un concepto fuerte de literalidad (1969, 1992). 
En nuestra exploración, donde los enunciados se presentaron sin contextos y se cauteló que expresaran variables del modelo ilocutivo y del modelo situacional de alto nivel para consejos, nuestro objetivo era obtener evidencia de que sus características construccionales ilocutivas, así aisladas, incidían en la valoración de su utilidad para la realización del acto de habla. Las valoraciones por defecto que obtuviéramos de los informantes nos permitirían mostrar la eficiencia de la noción de construcción ilocutiva para explicar la interpretación de expresiones consideradas indirectas en la tradición pragmática. Podríamos sostener, entonces, que la posibilidad de las oraciones de ser pragmáticamente adecuadas (de trasmitir con eficacia la fuerza ilocutiva) no depende exclusivamente del contexto en que son proferidas, como tendría que concluirse desde una lectura fuerte de la doctrina del significado como uso. Por el contrario, sería altamente plausible que una parte importante de esas posibilidades viniera integrada en las formas de que disponemos para trasmitir el sentido pragmático, teniendo siempre presente, por supuesto, que los parámetros de los modelos cognitivos ilocutivos que esas formas expresan solo se instancian plenamente en un contexto de habla.

Nuestra tesis surgió de los desarrollos construccionales en pragmática cognitiva que consideran los modelos cognitivos ilocutivos como base semántica para las operaciones de interpretación involucradas en la configuración del sentido de expresiones capaces de trasmitir un acto de habla específico, lo que ocurre, básicamente, porque estas expresiones proporcionan puntos de acceso metonímico relevantes a esa base. Excluyendo los detalles particulares de posibles situaciones concretas de uso, lo anterior quiere decir que las fórmulas ilocutivas realizan conjuntos de condiciones semánticas de modelos cognitivos ilocutivos (Del Campo, 2013), facilitando la producción e interpretación de actos de habla, y que tales condiciones establecen también un límite cognitivo a nuestras posibilidades expresivas. Los resultados, según se ha mostrado, confirman la hipótesis de que el valor construccional de los enunciados tiene consecuencias en la estimación de su sentido pragmático, lo que se verifica cuando este valor es separado de condiciones contextuales. Nuestra conclusión se basa en que las diferencias asociadas a las condiciones de construcción de sentido de los enunciados de la prueba (la operación de mecanismos cognitivos particulares) efectivamente mostraron relacionarse con diferencias en la calificación de su potencial de adecuación pragmática. Si, tal como se ha evidenciado, las expresiones tienen un valor construccional que nos dice algo acerca de su potencial de adecuación, entonces tenemos que aceptar que la producción y la interpretación ilocutivas no dependen exclusivamente del contexto y que parte de las condiciones destacadas del contexto mismo se hayan incorporadas en la forma. Desde el enfoque construccional, por tanto, los límites epistemológicos que impone una pragmática radical no son tales. En primer lugar, si las condiciones relevantes que definen las situaciones reiteradas del uso ilocutivo se organizan en subsistemas conceptuales como los modelos cognitivos idealizados, semántica y pragmática quedan enlazadas, pero sin el coste cognitivo del modelo pragmático convencionalista e inferencialista clásico. En segundo lugar, si las formas generadas por 
la instanciación de variables de modelos cognitivos ilocutivos pueden ser reutilizadas y elaboradas por los hablantes para transmitir un significado pragmático o recuperar el de las expresiones que interpretan, la creatividad lingüística queda salvaguardada sin que la pragmática deba renunciar a sus pretensiones explicativas.

\section{Bibliografía citada}

Baicchi, Annalisa, y Francisco Ruiz de Mendoza, 2010: "The cognitive grounding of illocutionary constructions within the theoretical perspective of the Lexical Constructional Model", Textus. English Studies in Italy 23 (3), 543-563.

Barcelona, Antonio, 2003: "The case for a metonymic basis of pragmatic inferencing: Evidence from jokes and funny anecdotes" en Klaus-Uwe PANTHER y Linda Thornburg (dirs.): Metonymy and Pragmatic Inferencing, Amsterdam/Philadelphia: John Benjamins Publishing Company, 81-102.

Croft, William, 2001: Radical Construction Grammar: Syntactic Theory in Typological Perspective, Oxford: Oxford University Press.

Del Campo, Nuria, 2012: "A constructional account of advising: Construal operations and social conventions", ATLANTIS. Journal of the Spanish Association of Anglo-American Studies $31(\mathrm{I}), 115-132$.

Del Campo, Nuria, 2013: Construcciones ilocutivas en inglés: un estudio desde el punto de vista del Modelo Léxico Construccional. Tesis de doctorado, Universidad de la Rioja: Servicio de Publicaciones, España.

Dummett, Michael, 1981: Frege: Philosophy of Language, London: Duckworth.

FIllmore, Charles, 1985: "Syntactic intrusions and the notion of grammatical construction", Proceedings of the Eleventh Annual Meeting of the Berkeley Linguistics 11, 73-86.

Fillmore, Charles, 1988: "The mechanisms of 'Construction Grammar', Proceedings of the Fourteenth Annual Meeting of the Berkeley Linguistics Society [BLS] 14, 35-55.

Fillmore, Charles, Paul Kay y Catherine O'Connor, 1988: "Regularity and Idiomaticity in Grammatical Constructions: The Case of Let Alone”, Language 64 (3), 501-538.

Goldberg, Adele, 1995: Constructions: A Construction Grammar Approach to Argument Structure, Chicago: Chicago University Press.

Goldberg, Adele, 2003: "Constructions: a new theoretical approach to language", TRENDS in Cognitive Sciences 7 (5), 219-224. 
Grice, Paul, 1975: "Logic and conversation” en Peter Cole y Jerry Morgan (dirs.): Syntax and semantics 3, New York: Academic Press, 41-58.

Johnson, Mark, 1991 [1987]: El cuerpo en la mente. Fundamentos corporales del significado, la imaginación y la razón, Madrid: Debate.

LAKOFF, George, 1987: Women, fire, and dangerous things. What categories reveal about the mind, Chicago, London: The University of Chicago Press.

LAKOFF, George, y Mark Johnson, 2004 [1980]: Metáforas de la vida cotidiana, Madrid: Cátedra.

LANGACKER, Ronald, 1987: Foundations of Cognitive Grammar: Theoretical Prerequisites, Stanford: Stanford University Press.

LeECH, Geoffrey, 1997 [1983]: Principios de pragmática, Universidad de la Rioja: Servicio de Publicaciones.

Panther, Klaus-Uwe, y Linda Thornburg, 2007: "Metonymy" en Dirk GeeraerTs y Hubert Cuyckens (dirs.): The Oxford Handbook of Cognitive Linguistics, Oxford: University Press, 236-263.

Pérez Hernández, Lorena, 2001: Illocution and Cognition. A Constructional Approach, Universidad de la Rioja: Servicio de Publicaciones.

Pérez Hernández, Lorena, y Francisco Ruiz de Mendoza, 2002: "Grounding, semantic motivation, and conceptual interaction in indirect directive speech acts", Journal of Pragmatics 34, 259-284.

Pérez Hernández, Lorena, y Francisco Ruiz de Mendoza, 2011: "A lexical constructional model account of illocutions", VIAL 8, 99-139.

Rivano, Emilio, 2009: "Wittgenstein y Chomsky en contrapunto: Notas epistemológicas y metodológicas en torno al lenguaje, conocimiento y comunicación”, Filología y Lingüística XXXV (2), 207-232.

Ruiz de Mendoza, Francisco, 2007: "High-level cognitive models: search of a unified framework for inferential and grammatical behaviour" en Krzysztof Koseckı (dir.): Perspectives on metonymy, Frankfurt/Main: Peter Lang, 11-30.

Ruiz de Mendoza, Francisco, 2011: "Metonymy and cognitive operations" en Reka Benczes, Antonio Barcelona y Francisco Ruiz de Mendoza (dirs.): Defining metonymy in cognitive linguistics: towards a consensus view, Amsterdam/Philadelphia: John Benjamins, 103-124.

Ruiz de MendozA, Francisco, y Annalisa Baicchl, 2007: "Illocutionary constructions: cognitive motivation and linguistic realization" en Istvan Kecskes y Laurence Horn (dirs.): Explorations in Pragmatics: Linguistic, Cognitive, and Intercultural Aspects, Berlin / New York: Mouton de Gruyter, 95-128. 
Ruiz de Mendoza, Francisco, y Nuria del Campo, 2012: "La lingüística cognitiva y la pragmática” en Iraide IbarRetXe-Antuñano y Javier Valenzuela (dirs.): Lingüística Cognitiva, Barcelona: Anthropos, 329-347.

Ruiz de Mendoza, Francisco, y Alicia Galera, 2012: "Modelos cognitivos, operaciones cognitivas y usos figurados del lenguaje", Forma y Función 25 (2), 11-38.

Ruiz de Mendoza, Francisco, y Francisco GonzÁlvez, 2010: "Illocutionary meaning revisited: subjective-transitive constructions in the Lexical-Constructional Model" en Piotr StaLmaszczyk (dir.): Cognitive and Phenomenological Turns in Philosophy of Language and Linguistics, Frankfurt am Main: Peter Lang Verlag, 65-77.

Ruiz de Mendoza, Francisco, y Ricardo Mairal, 2008: "Levels of description and constraining factors in meaning construction: an introduction to the Lexical Constructional Model", Folia Linguistica 42 (2), 355-400.

Schank, Roger, y Robert Abelson, 1977: Scripts, plans, goals and understanding: an inquiry into human knowledge structures, Hilsdale, NJ: Erlbaum.

SeARLE, John, 1969: Speech acts. An essay in the philosophy of language, Cambridge: University Press.

Searle, John, 1975: "Indirect speech acts" en Peter Cole y Jerry Morgan (dirs.): Syntax and semantics 3, New York: Academic Press, 59-82.

SeARLE, John, 1992: The rediscovery of the mind, Cambridge: Cambridge University Press.

Thornburg, Linda, y Klaus-Uwe Panther, 1997: "Speech act metonymies" en Wold-Andreas Liebert, Gisela ReDeker y Linda Waugh (dirs.): Discourse and perspectives in cognitive linguistics, Amsterdam and Philadelphia: Benjamins, 205-219.

Tomassını, Alejandro, 1988: El pensamiento del último Wittgenstein, México: Trillas.

WINCH, Meter, 1958: The idea of a social science and its relation to philosophy, London: Routledge and Kegan Paul.

Wittgenstein, Ludwig, 1953: Philosophical investigations, New York: Macmillan. 